IP Periodica Polytechnica

Mechanical Engineering

57(2), pp. 47451 2013

DOI: 10.3311/PPme.7044

Creative Commons Attribution (1)

RESEARCH ARTICLE

\section{Application of alcohol vapour as atomizing fluid in a micro gas turbine burner}

\author{
Attila Kun-Balog, Krisztián Sztankó
}

Received 2013-05-29, accepted 2013-09-03

\begin{abstract}
Liquid fuels have to be atomized before combustion. In micro gas turbines twin-fluid atomizers are widely used for this purpose. In this type of atomizers generally high velocity air flow disintegrates the liquid fuel stream into droplets, but superheated vapour can also be applied as atomizing fluid. In our experiments a burner of a Capstone C30 micro gas turbine was used, which was built into a test rig developed by us. During measurements diesel oil was used as liquid fuel. Atomizing fluids were air and aqueous ethyl alcohol vapour at different pressures. In every combustion experiment, emissions of carbon monoxide, unburned hydrocarbons and nitrogen oxides were measured. The results showed that application of alcohol vapour as atomizing fluid instead of air improves the quality of combustion. Increasing the atomizing pressure up to a defined optimum value also reduces emissions significantly.
\end{abstract}

\section{Keywords \\ micro gas turbine $\cdot$ atomizing $\cdot$ alcohol vapour}

\section{Acknowledgement}

The work reported in the paper has been developed in the framework of the project "Talent care and cultivation in the scientific workshops of BME”. This project is supported by the grant TÁMOP - 4.2.2.B-10/1-2010-0009.

\section{Attila Kun-Balog}

Department of Energy Engineering at Budapest University of Technology and Economics, H-1111 Budapest, Múegyetem rkp. 3-9, Hungary

e-mail: kunbalog@energia.bme.hu

\section{Krisztián Sztankó}

Department of Energy Engineering at Budapest University of Technology and Economics, H-1111 Budapest, Múegyetem rkp. 3-9, Hungary

e-mail: sztanko@energia.bme.hu

\section{Introduction}

Micro gas turbines operating with liquid fuels are designed for using diesel oil and kerosene. The manufacturers recommend biodiesel and biodiesel-alcohol (max. $20 \%$ ethanol or methanol) mixture as renewable fuel. These can be applied without any modification of the fuel system of gas turbines, and their applications do not cause any noticeable decrease in power or efficiency [1]. In case of application of renewable fuels, different from the above mentioned ones, their physical, chemical and combustion properties have to be examined. Required modifications of the fuel system can be designed considering these properties.

Utilization of vegetable oil and biodiesel fuels in micro gas turbines has already been examined by several researchers [2, 3], but only few experiments are known for using alcohols in gas turbines [4]. Ethanol has advantageous combustion properties, but it is not widespread in energy generation because of its high production cost.

There are several processes to produce alcohols (mainly aqueous solutions of ethanol) for combustion purpose. Raw materials may be materials of plant origin containing carbohydrate, starch or cellulose. The difference is in their price and the energy demand of the required processing technology. It is a longstanding debate if it is possible to produce ethanol with positive energy balance. It is clear, that the production of anhydrous pure ethanol is the most expensive, and therefore the application of equipment, in which alcohol of lower concentration can also be utilized economically, is preferred. Then the energy demand of distillation applied during the alcohol production can be reduced, dehydration can be omitted, thus the energy balance improves [5].

Utilization of alcohols as fuel in heat engines designed for conventional fuels hits against a lot of difficulties. Ethanol has low lubricity and strong corrosion effects on metals in the presence of a small amount of water, and attacks a certain part of the materials used for sealing. Its net heating value is lower than that of the diesel oil's or kerosene's, therefore the delivery capacity of the originally built-in fuel pump of heat engines usually proves to be low. Under the same conditions more complete and 
perfect combustion can be ensured with pure alcohol, than conventional liquid fuels mentioned before. But if aqueous alcohol is supplied to the combustion chamber in liquid state by itself or mixed with another fuel as a spray, combustion occurs in more adverse conditions because of high evaporation heat of watercontent. If aqueous alcohol is supplied to the reaction zone after evaporating, this problem does not occur.

\section{Experimental methods}

\subsection{The micro gas turbine burner}

A burner of a Capstone C30 recuperated micro gas turbine (Fig. 1) was used for the experiments. This burner works with a pneumatic (twin fluid) atomizer, in which compressed air, taken from the compressor of the gas turbine, at $150-200^{\circ} \mathrm{C}$ makes the atomization. This air accelerates during its expansion in the nozzle of the atomizer, and disintegrates the liquid fuel jet into droplets. Combustion air heated in the internal recuperator enters the mixing tube of the burner through radial-tangential orifices, where the air mixes with the atomized fuel in a swirling flow. Fuel droplets evaporate in the mixing tube, so a mixture of fuel vapour and air leaves the tube. In the ideal case combustion is homogenous, and it starts after exiting the mixing tube.

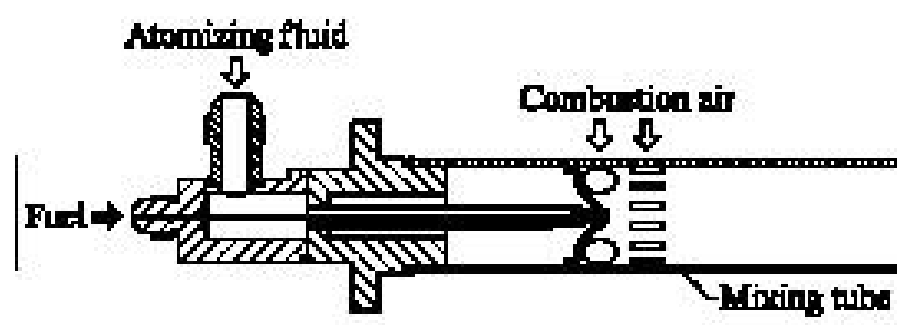

Fig. 1. Burner of Capstone $\mathrm{C} 30$ micro gas turbine

In a micro gas turbine the pressure difference between compressor bleed air used for atomization and the combustion chamber is equal with the pressure drop of the internal recuperator (approximately 0,2-0,3 bar). Since this pressure drop reduces the efficiency of the gas turbine, manufacturers apply recuperators with low pressure drop. As built-in burners use this low pressure difference to accelerate the atomizing fluid, the quality of the atomization does not live up to the expected level based on construction and working principle of the atomizer. In order to maintain higher quality atomization the increase of atomizing pressure would be preferable. This requirement could be satisfied by an auxiliary compressor. The energy consumption of this compressor would increase the electrical self-consumption of the micro gas turbine, so it would decrease the efficiency of the turbine.

Another solution for increasing the atomizing pressure is ensured by changing the atomizing air with superheated vapour. In an appropriate heat exchanger high-pressure superheated vapour can be produced by hot exhaust gas $\left(275^{\circ} \mathrm{C}\right)$. Vapour accelerates to high velocity expanding in the atomizer ensuring excellent atomization quality. Vapour can be produced by evaporation and superheating of water, aqueous ethanol or pure ethanol. A liquid pump supplies the heat exchanger, whose electrical consumption is negligible compared to the consumption of the auxiliary compressor mentioned above.

\subsection{Combustion test rig}

Considering probable experiments in the future examination of a separated burner of the micro gas turbine seemed to be expedient. So a combustion test rig (Fig. 2) had to be built, in which the burner is capable to operate with different atomizing fluids at elevated pressure.

Superheated vapour used as atomizing fluid was produced in an own developed, electrically heated tubular vapour generator. The atomizing pressure was controlled by the mass flow rate of the evaporated fluid. In case of atomizing by air the compressed air system of our laboratory was used with a pressure regulator. Combustion air was supplied by a centrifugal fan to the electrical air preheater, where it was heated up to the required temperature $\left(250-400^{\circ} \mathrm{C}\right)$.

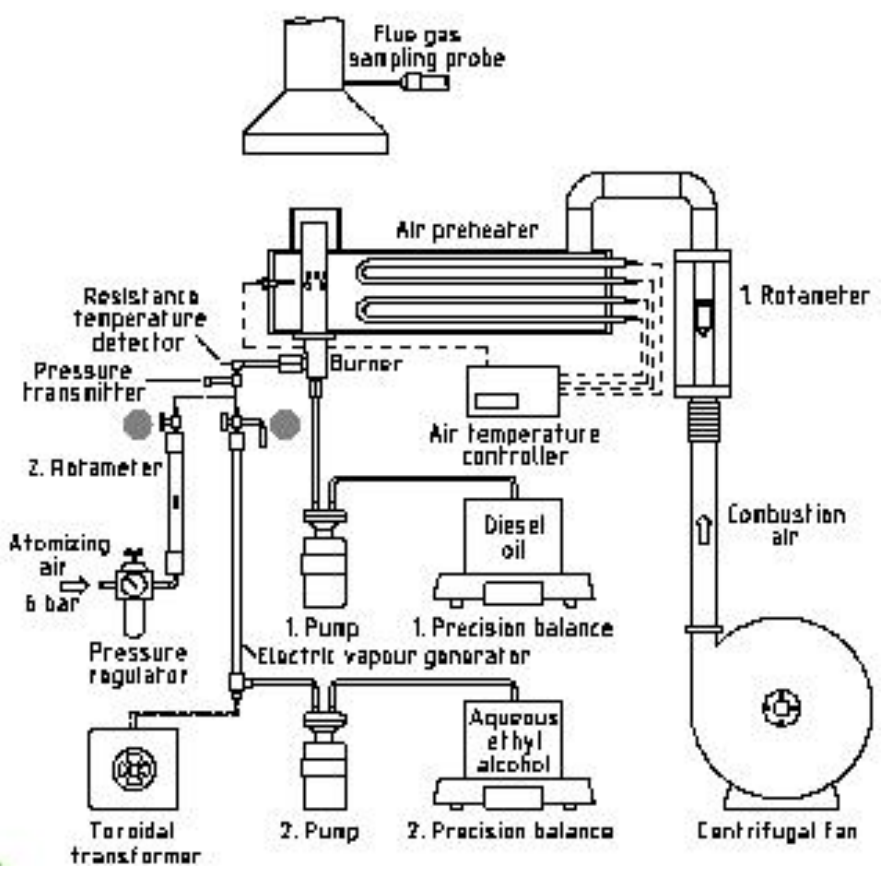

Fig. 2. Combustion test rig, I. Atomizing by air, II. Atomizing by superheated vapour

As there was no possibility to build a pressure-tight combustion chamber with visual accessibility, so the burner was examined just under atmospheric conditions. Although the measuring conditions were different from the operating ones, future tests on the micro gas turbine will verify with high probability that the conclusions extracted from measurement results are appropriate.

\subsection{Measuring procedure}

During combustion experiments with air as atomizing fluid the pressure, temperature and volume flow rate of the combustion and atomizing air and the mass flow rate of the fuel were continuously measured. At experiments with aqueous ethyl al- 
cohol the mass flow rate of the evaporated medium, the electrical power consumption of the vapour generator, and the pressure and temperature of atomizing vapour were measured. The temperature of vapour was $180^{\circ} \mathrm{C}$ at every measurement, and the temperature controller of the combustion air preheater was set to $250^{\circ} \mathrm{C}$. Heating the atomizing air was not possible, so it was $20^{\circ} \mathrm{C}$ during measurements. The combustion products were conducted to a flue-gas channel with the help of a flue gas collector, and the samples taken from here were examined by a Horiba MEXA-8120F flue-gas analyzer. Electronic data collecting was applied for all measured parameters except volume flow rates.

Measurements were carried out with diesel oil (according to EN 590: 2009 standard) at $15 \mathrm{~kW}$ firing power, air and superheated vapour produced from ethanol-water mixture $(55 \mathrm{v} / \mathrm{v} \%)$ were used as atomizing fluid at different atomizing pressures. Since the mass flow rate of atomizing air increased by raising its pressure, so the mass flow rate of combustion air supplied by the centrifugal fan had to be reduced in order to keep up the constant $(1,2)$ air/fuel equivalence ratio. Using alcohol vapour as atomizing fluid the mass flow rate of diesel oil had to be reduced in order to keep up the constant firing power, considering the heating value of ethanol-water mixture.

\section{Results and discussion}

\subsection{Atomization}

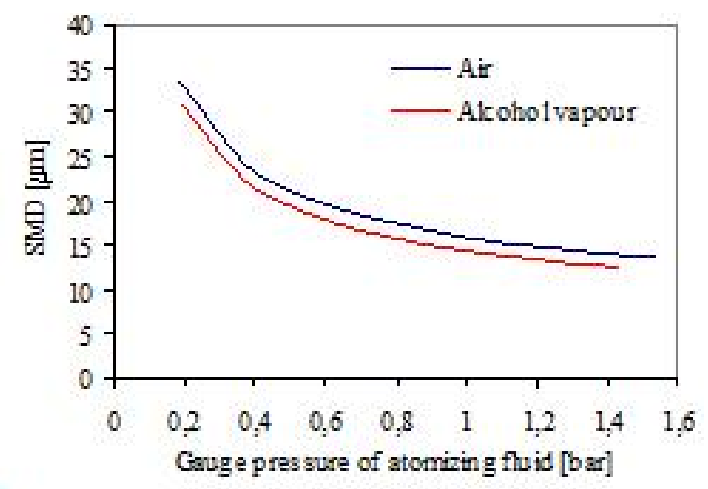

Fig. 3. Sauter mean diameter of fuel drops

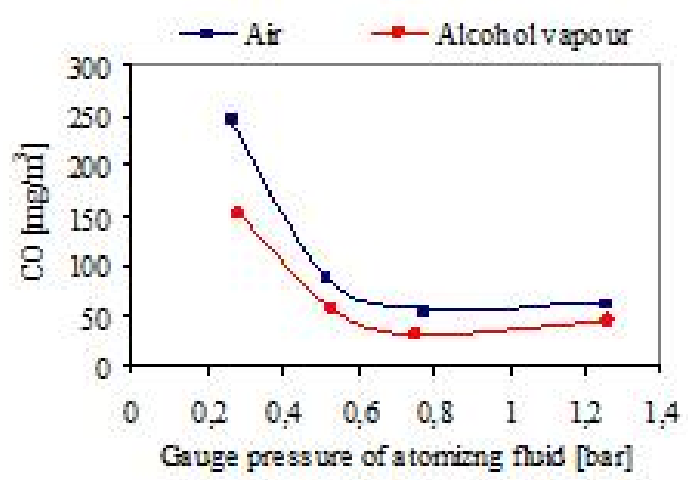

Fig. 4. Carbon monoxide

The size of droplets produced by the twin fluid atomizer of Capstone C30 micro gas turbine burner mainly depends on the

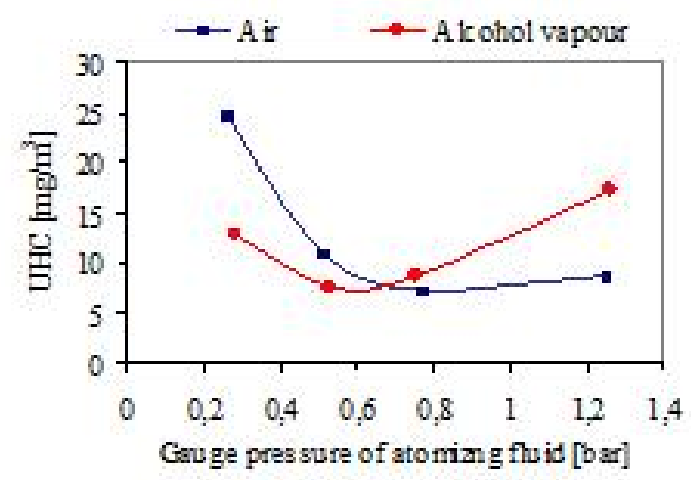

Fig. 5. Unburned hydrocarbon

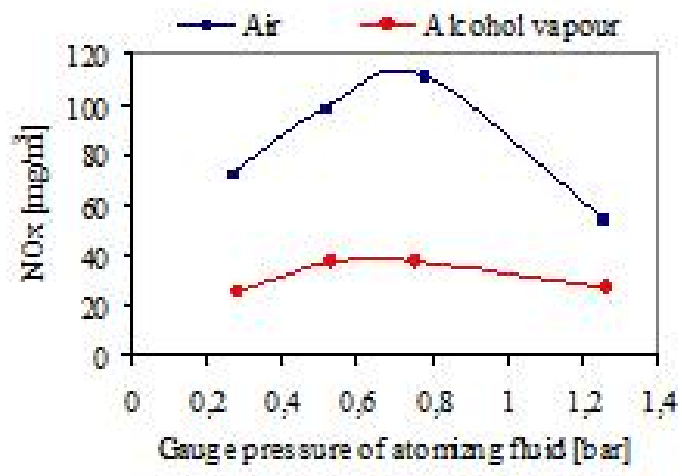

Fig. 6. Nitrogen oxides

ratio of the mass flow rate of liquid fuel and atomizing fluid. Overpressure and temperature, which can be measured in the atomizing fluid before entering the burner, clearly define the mass flow rate of the atomizing fluid effusing through the nozzle. Tab. 1 shows the measured mass flow rates of diesel oil, air and aqueous alcohol vapour at different pressures of atomizing fluid.

For estimation of the volume-surface (Sauter) mean diameter of atomized fuel droplets the following equation can be used (it was created by Lefebvre and Rizk [6] for an atomizer of the same type).

$$
\begin{aligned}
d_{23}=S M D=0, & 48 \cdot D_{0}^{0,6} \cdot\left(\frac{\sigma_{L}}{w_{A}^{2} \cdot \rho_{A}}\right)^{0,4} \cdot\left(1+\frac{\dot{m}_{L}}{\dot{m}_{A}}\right)^{0,4} \\
& +0,15 \cdot\left(\mu_{L}^{2} \cdot \frac{D_{0}}{\sigma_{L} \cdot \rho_{L}}\right)^{0,5} \cdot\left(1+\frac{\dot{m}_{L}}{\dot{m}_{A}}\right)
\end{aligned}
$$

In the equation $D_{0}[\mathrm{~m}]$ is the nozzle diameter, $w_{A}[\mathrm{~m} / \mathrm{s}]$ is the air velocity, $\dot{m}_{L}[\mathrm{~kg} / \mathrm{s}]$ and $\dot{m}_{A}[\mathrm{~kg} / \mathrm{s}]$ are the mass flow rates of liquid and air, $\sigma_{L}(0,025 \mathrm{~N} / \mathrm{m}), \mu_{L}(0,002$ Pas $)$ and $\rho_{L}\left(835 \mathrm{~kg} / \mathrm{m}^{3}\right)$ are the surface tension, dynamic viscosity and the density of the liquid. Substituting our measured results and physical properties of mediums into the equation we get the following diagram.

The size of atomized fuel droplets can be reduced with the application of alcohol vapour. However, the reason of this is reducing the mass flow rate of diesel oil, since that was substituted with alcohol. Mean diameter was halved by increasing the atomizing pressure from 0,25 to 1,25 bar with both atomizing fluids. Pressure difference, which accelerates the atomizing fluid, 
Tab. 1. Mass flow rates of fuel and atomizing fluids at different pressures

\begin{tabular}{cccccc}
\hline Atomizing fluid & Mass flow rate [g/s] & \multicolumn{4}{c}{ Gauge pressure of atomizing fluid [bar] } \\
& & 0,25 & 0,5 & 0,75 & 1,25 \\
\hline \multirow{2}{*}{ Air } & Diesel oil & 0,35 & 0,35 & 0,35 & 0,35 \\
& Air & 0,23 & 0,32 & 0,39 & 0,51 \\
Alcohol vapour & Diesel oil & 0,29 & 0,28 & 0,26 & 0,23 \\
& Alcohol vapour & 0,21 & 0,25 & 0,31 & 0,42 \\
\hline
\end{tabular}

reaches a critical value at highest pressure shown on Fig. 3, and the atomizing fluid exits the nozzle at sonic speed. Above this value exit velocity can not be increased further, just the mass flow rate; and so mean droplet diameter can not be increased further effectively.

\subsection{Flue gas analysis}

Effects of atomizing pressure increase and changeover from air to alcohol vapour as atomizing fluid on combustion quality were examined by flue gas analysis. During combustion experiments concentration of carbon monoxide ( $\mathrm{CO})$, unburned hydrocarbons (UHC) and nitrogen oxides $\left(\mathrm{NO}_{\mathrm{x}}\right)$ were measured in the flue gas. The following three figures sum up the results.

During air blast atomization when the gauge pressure of the atomizing air was increased to $0,6-0,7$ bar the emission of carbon monoxide and unburned hydrocarbons decreased. Further increase in pressure caused low-rate increase in the concentration of these air pollutants. As it was shown earlier increase in the pressure of atomizing fluid reduces the volume-surface mean diameter of droplets. Heating up and evaporating of smaller fuel droplets take place in shorter time, so a better mixing with air can be realized in the mixing tube, thereby a more homogenous vapour-air mixture gets to the reaction zone. Certainly it ensures more and more complete and perfect combustion, which causes a decrease of the carbon monoxide and unburned hydrocarbons emissions. However the increase in pressure results less and less droplet size reduction at high pressures, but at the same time exit velocity of atomizing fluid reaches the local speed of sound. At this flow velocity the residence time of fuel fractions for evaporating, igniting and complete burning decreases, so emissions of carbon monoxide and unburned hydrocarbons increase, as it was shown by the measurement results.

Diesel oil contain a negligibly small amount of nitrogen, so during combustion nitrogen oxides generate mainly from the nitrogen content of air in thermal way in the flame front. The highest temperature occurs when conditions of combustion are the most advantageous. Increase in pressure of atomizing air causes rising in emission of nitrogen oxides at first because of the improvement of combustion quality, and then emission reaches a maximum. This maximum is at the minimum of carbon monoxide and unburned hydrocarbon emissions. By further increase of pressure raises average velocity of mixture. Hence residence time of nitrogen content of air decreases in the flame front, so the formation of nitrogen oxides drops.
As it was mentioned earlier in case of atomizing by alcohol vapour it was possible to reduce the mass flow rate of diesel oil beside conservation of constant firing power. In consequence using alcohol vapour stream results smaller fuel drops than air stream at the same pressure, so the concentration of carbon monoxide decreased in the flue gas. Emission of unburned hydrocarbons also decreased but just below 0,6 bar atomizing pressure. Since ignition temperature of alcohol is higher, and its ignition delay is longer than that of diesel oil's, therefore the time for heating up and igniting is longer, so necessary residence time for the complete burning out is longer than at atomizing by air. On the contrary, residence time shortens at higher pressure, therefore emission of unburned hydrocarbon increase is stronger. Emission of carbon monoxide increases slightly after reaching the minimal value, because the water-content of atomizing fluid has a catalytic effect of on the combustion of carbon monoxide.

The emission of nitrogen oxides was significantly smaller than that measured at atomizing by air because of the flame cooling effect of water-content of aqueous alcohol and because ethyl alcohol has lower burning temperature than diesel oil. Emission of nitrogen oxides had a maximum value, but differences between measured values were much smaller than at atomizing by air.

It can be stated different atomizing fluids have different optimal gauge pressure for low pollutant emissions. In case of air it would be expedient to increase pressure to the critical value; it would be optimal for all of examined flue gas components. In case of atomizing by alcohol vapour the optimal gauge pressure is between 0,6 and 0,7 , where emission of carbon monoxide and unburned hydrocarbons is also minimal, but it is true that the emission of nitrogen oxides reaches its maximum here. Although increase in pressure reduces emission of nitrogen oxides slightly, but it increases the volume of unburned hydrocarbons significantly.

In Capstone C30 micro gas turbine the gauge pressure of atomizing air is 0,2-0,3 bar in normal operating state, which is far from the optimal value defined above for atomizing by air at atmospheric conditions, so expectedly emissions are also far from accessible minimal levels. 


\section{Conclusions}

After the analysis of measurement results we found that it would be expedient to operate the burner of Capstone C30 micro gas turbine with higher pressure atomizing fluid for lower pollutant emission. At atomization by air exit velocity has to be increased to sound velocity by the increase of atomizing pressure for minimal emission. Higher quality of atomization can be achieved by the application of alcohol vapour as atomizing fluid at lower optimal pressure defined above.

At micro gas turbines supplying the vaporizable fuels in vapour state instead of liquid state to the combustion chamber contributes to improve the overall efficiency. By this solution the heat demand of evaporation is covered by heat not from the combustion chamber but from the flue gas. It is particularly important, if utilized alcohol has high water-content. If we use it in liquid state mixing with diesel oil as emulsion, pollutant emissions will increase, as it was verified by former researches [8]. But substituting of diesel oil for alcohol with high water-content in vapour state improves the quality of combustion, as it was verifiable from measurement results.

\section{References}

1 Capstone MicroTurbine-Application Guide: Liquid Biofuel Requirements, www.microturbine.com

2 Bolszo C, McDonell V, Emissions optimization of a biodiesel fired gas turbine, Proceedings of the Combustion Institute, 32(2), (2009), 2949-2956, DOI $10.1016 /$ j.proci.2008.07.042

3 Panchasara H, Simmons B, Agrawal A, Spear SK, Daly DT, Combustion Performance of Biodiesel and Diesel-Vegetable Oil Blends in a Simulated Gas Turbine Burner, Journal of Engineering for Gas Turbines and Power, 131(3), (2009), 031503, DOI 10.1115/1.2982137

4 Moliere M, Vierling M, Aboujabib M, Patil P, Eranki A, Campbell A, Trivedi R, Nainani A, Roy S, Pandey N, Gas turbines in alternative fuel applications: Bio-ethanol field test, In: Volume 1: Aircraft Engine; Ceramics; Coal, Biomass and Alternative Fuels; Controls, Diagnostics and Instrumentation; Education; Electric Power; Awards and Honors, June 8-12, 2009, Orlando, Florida, USA, pp. 341-348, DOI 10.1115/GT2009-59047

5 Saxena S, Schneider S, Aceves S, Dibble R, Wet ethanol in HCCI engines with exhaust heat recovery to improve the energy balance of ethanol fuels, Applied Energy 98, 98, (2012), 448-457, DOI 10.1016/j.apenergy.2012.04.007

6 Lefebre A, Ballal D, Gas turbine combustion, Taylor and Francis Group, 2010.

7 Williams A, Combustion of liquid fuel sprays, Butterworths \& Co, London, 1990.

8 Sztanko K, Kun-Balog A, Applicability of bioethanol-water mixtures in micro gas turbines, Austrian-Croatian-Hungarian Combustion Meeting ACH2012, (12-13 April 2012). 